\title{
Diseño teórico y simulación de un relevador de sobrecorriente con capacidades dinámicas de ajuste
}

\section{Theoretical Design and Simulation of an Overcurrent Relay with Dynamic Setting}

\author{
Conde-Enriquez A. \\ Facultad de Ingeniería Mecánica y Eléctrica. Universidad Autónoma de Nuevo León. \\ San Nicolás de los Garza, Nuevo León, México. \\ E-mail:con_de@yahoo.com \\ Vázquez-Martínez E. \\ Facultad de Ingeniería Mecánica y Eléctrica. Universidad Autónoma de Nuevo León. \\ San Nicolás de los Garza, Nuevo León, México. \\ E-mail:evazquez@gama.fime.uanl.mx \\ Cantú-Gutiérrez V.P. \\ Facultad de Ingeniería Mecánica y Eléctrica. Universidad Autónoma de Nuevo León. \\ San Nicolás de los Garza, Nuevo León, México. \\ E-mail:vcantugtz@yahoo.com
}

(Recibido: junio de 2007; aceptado: febrero de 2008)

\section{Resumen}

En este artículo recomendamos una nueva lógica funcional para un relevador de sobrecorriente de tiempo inverso. Este relevador tiene mayor sensibilidad y tiempos de operación de respaldo reducidos, comparados con relevadores convencionales; la información requerida por el relevador propuesto es determinada únicamente en la localidad del relevador. Se presenta el diagrama funcional del relevador, describiendo cada uno de sus bloques funcionales. El desempeño del relevador propuesto es comparado con relevadores de sobrecorriente convencionales y relevadores de sobrecorriente de se cuencianegativa.

Descriptores: relevador de sobrecorriente, relevador adaptivo, corriente de arranque, sensibilidad, curva de tiempo, coordinación.

\begin{abstract}
This paper presents a new logic of operation for time overcurrent relays. With the application of the proposed technique, the sensitivity of time overcurrent relays is increased. We recommend a new coordination system for time overcurrent relays too. The purpose of the coordination process is to find a time element function that allows it to operate using a constant back-up time delay, for any fault current. The results obtained from time overcurrent adaptive relays are collated, and the results from analysis of negative sequence relays and conventional time overcurrent relays are also presented.
\end{abstract}

Keywords: Overcurrent relay, adaptive relay, pick-up current, sensitivity, time curve, coordination. 
DOI: http://dx.doi.org/10.22201/fi.25940732e.2010.11n2.013

Diseño teórico y simulación de un relevador de sobrecorriente con capacidades dinámicas de ajuste

\section{Introducción}

La aplicación de relevadores de sobrecorriente de tiempo inverso en redes eléctricas presenta serias limitaciones de sensibilidad y tiempos elevados de operación para corrientes mínimas de fallas. El incremento desproporcionado de la densidad de carga y la escasa construcción de líneas de sub-transmisión y distribución, provoca que los sistemas eléctricos sean sometidos a condiciones más severas de carga. El ajuste de un relevador de sobrecorriente es mayormente comprometido, debido a que los valores mínimos de corriente de falla y ajuste del relevador son comparables, dificultando la correcta detección de la falla. Una falla en condiciones de mínima demanda representa una aportación menor de corriente; es precisamente en esta condición cuando se requiere mayor sensibilidad en la protección. Sin embargo, el ajuste de la corriente de arranque del relevador es efectuado usando los valores máximos de corriente de carga (pocos minutos por día) y en las configuraciones críticas de la red en donde el relevador pueda disparar por carga, como en operaciones de transferencia de potencia (esquemas de emergencia). Entonces, el ajuste de la corriente de arranque es establecido durante escenarios poco frecuentes o de corta duración, teniendo como resultado una insensibilización mayor de la protección.

Otra limitación de la protección de sobrecorriente son los elevados tiempos de respaldo para fallas no máximas. El criterio de coordinación se determina para los valores máximos de corriente de falla (3-5\% del total de fallas) y durante condiciones máximas de demanda (solo unos cuantos minutos al día), esto debido a la convergencia de las curvas de tiempo para valores mayores de corriente.

Para las fallas restantes, que son las más frecuentes, el tiempo de operación del relevador es mayor. Este comportamiento es propio de los relevadores de sobrecorriente y se ha comprobado que es muy adecuado para la protección de sistemas eléctricos en donde es frecuente y temporal su operación sobre los valores nominales. Esta situación no es tan conveniente cuando se presenta en la protección de respaldo; por la naturaleza misma del relevador de sobrecorriente resultan elevados tiempos de operación, sometiendo al sistema a tolerar corrientes que provocan esfuerzos térmicos y mecánicos que pudieran evitarse. La limitación de tiempo es acentuada cuando el dispositivo primario tiene una curva de tiempo con diferente grado de inversión que la del relevador de respaldo.

En trabajos recientes (Askarian et al., 2003), (Urdaneta et al., 1988) y (Abdelaziz et al., 2002), se proponen diferentes métodos de coordinación; todos son dependientes de canales de comunicación para actualizar los ajustes, por lo que es necesario considerar los factores económicos involucrados.

En redes aisladas (rurales) o en redes altamente interconectadas en donde no es viable implementar una estrategia mediante canales de comunicación, es posible realizar el ajuste automático del relevador utilizando la corriente local e información fuera de línea del dispositivo de protección primario.

El relevador propuesto no requiere de algún medio físico de comunicación. El proceso de coordinación es automático e independiente de futuros cambios en el sistema (tales como cambios topológicos, de generación y carga).

En este trabajo se propone un relevador adaptivo de sobrecorriente de tiempo inverso. Este criterio de protección de fase puede ser aplicado tanto en sistemas de potencia como en sistemas industriales. Los objetivos principales del relevador adaptivo son: incrementar la sensibilidad del relevador para corrientes mínimas de falla durante condiciones de baja demanda. El segundo es determinar una función de tiempo que asegure la operación de respaldo con un retardo constante de tiempo relativo al dispositivo primario para cualquier valor de corriente de falla.

\section{Los beneficios del relevador propuesto son:}

- La corriente de arranque depende de la magnitud de la corriente de carga, resultando en mayor sensibilidad de la protección cuando más lo requiere.

- El tiempo de respaldo es independiente de la magnitud de la corriente de falla, resultando en menor tiempo de respaldo que en relevadores convencionales.

El proceso de diseño de un equipo o sistema digital destinado a operar en tiempo real, consta de dos etapas básicas. La primera incluye la investigación y desarrollo de los algoritmos y su evaluación por simulación digital. La segunda consiste en el desarrollo de un prototipo del equipo o sistema y su prueba en condiciones de laboratorio, posteriormente, en condiciones reales de operación. La única infraestructura requerida para la primera etapa es el equipo de cómputo, dotado del "software" apropiado para la aplicación.

En la segunda etapa se requiere contar con un laboratorio que permita simular físicamente el sistema real al que está destinado el equipo, y tener facilidades para el diseño y construcción del prototipo. El simulador 
físico puede ser sustituido o complementado por un simulador digital en algunas aplicaciones, pero la construcción del prototipo es inevitable. Cuando el equipo a diseñar es un relevador digital de protección de sistemas eléctricos de potencia, la construcción del prototipo es una labor muy especializada, que no siempre está al alcance de las instituciones de investigación.

En este trabajo se presenta el desarrollo de un algoritmo de protección que permita mejorar el funcionamiento de un relevador de sobrecorriente realizando modificaciones a nivel de "firmware" del relevador, entonces es asumido que la adecuación del hardware necesario y del conjunto de pruebas es un problema resuelto por los fabricantes de equipo de protección.

Los resultados presentados fueron obtenidos mediante la simulación de los algoritmos de diseño del relevador, programados en paquetes profesionales, sin realizar la construcción de un prototipo físico. Sin embargo, los resultados obtenidos respaldan los fundamentos teóricos planteados y, cumpliéndose las condiciones establecidas, las futuras pruebas en condiciones reales de operación.

\section{Limitaciones del relevador de sobrecorriente}

La protección de sobrecorriente usa la corriente como único indicador de la ubicación de la falla. Sin embargo, la corriente de falla depende del voltaje de pre-falla y la impedancia de Thevenin en el punto de falla. Esto provoca que el relevador de sobrecorriente tenga un alcance dinámico, dependiente de la magnitud de la corriente de falla, presentando menor sensibilidad durante mínima demanda y en fallas bifásicas. Por otra parte, la corriente máxima de carga puede ser similar en magnitud a la corriente mínima de falla; esto dificulta la correcta discriminación entre el estado normal de operación y la condición de falla.

Otro problema en la protección de sobrecorriente son los elevados tiempos de respaldo para corrientes mínimas de falla; esta limitación es originada por la corriente de carga (altos valores de arranque) y la divergencia natural de las características de operación de los relevadores. También, cuando las protecciones primaria y de respaldo tienen tipos de curvas diferentes, es difícil conseguir una coordinación adecuada.

Una solución para las limitaciones de relevadores de sobrecorriente es usar criterios adaptivos. En este artículo, se propone una estructura de un relevador adaptivo de sobrecorriente. Este relevador adaptivo es compuesto de dos elementos adaptivos: corriente adaptiva de arranque y tiempo adaptivo de operación.

\section{Corriente adaptiva de arranque}

Se propone un ajuste dinámico de la corriente de arranque en función de la demanda de corriente:

$$
I_{a}=\frac{1}{N} \sum_{j=1}^{N}\left(I_{k}\right)_{j}+\Delta I,
$$

donde $I_{k}$ es la corriente en estado estable del sistema eléctrico, $\Delta I$ representa un margen de seguridad con un valor propuesto de $15 \%$ del valor máximo de la corriente de carga, y $N$ debe ser seleccionado de tal manera que el intervalo $N x \Delta t$ (periodo de muestreo) tenga una duración de entre uno y varios minutos, similar a los integradores de demanda usados en medidores eléctricos.

La ecuación (1) asegura que el relevador tenga en todo momento la corriente de arranque mínima necesaria para evitar una operación incorrecta debido al efecto de la carga. Esto provee mayor sensibilidad, porque el valor de $I_{a}$ es también pequeño durante condiciones mínimas de carga.

La lógica de control de la corriente de arranque tiene la tarea de mantener constante $I_{a}$ durante una falla. Si la línea es desenergizada, la lógica de control asigna un valor máximo $I_{a}^{\max }$, el cual puede ser similar al ajuste de un relevador convencional en la misma ubicación del adaptivo. Durante un intervalo completo de demanda, el valor $I_{a}$ es dado en el relevador al final del intervalo previo de demanda. La acción de filtrado pasa-bajos inherente en el concepto de demanda simplifica la lógica del relevador adaptivo.

Durante incrementos súbitos de la corriente de carga (transferencia de potencia o conexión de carga), el relevador tiene una lógica de detección de falla en orden de supervisar $I_{a}$. Esta lógica incluye una verificación de la corriente de secuencia negativa y secuencia positiva, ambos combinados en una lógica $O R$. El detector de secuencia negativa es propuesto para detectar fallas bifásicas (Elneweihi et al., 1992). En redes de bajo voltaje, la corriente de secuencia negativa originada por fallas bifásicas es mayor que la corriente de secuencia negativa originada por desbalances, permitiendo un buen ajuste. El detector de secuencia positiva es propuesto para detectar fallas trifásicas, este tiene el mismo ajuste que un relevador convencional de fase. Por tanto, esta lógica discrimina entre incrementos súbitos de carga y fallas (simétricas o asimétricas).

El desempeño de la lógica de detección de falla es analizada en la red eléctrica de la figura 1a. La secuencia de operación simulada consiste en tres escenarios: desbalance en estado estable, cortocircuito bifásico en la 
línea adyacente al Rele $A$ y disparo de la línea fallada (transferencia de potencia). Se analiza el desempeño del Rele A; este relevador debe tolerar la condición de desbalance, respaldar la falla en la línea adyacente, y no dispara para transferencia de potencia. Durante el desbalance severo en estado estable ( $25 \%$ según ANSI/IEEE Std 141-1986, 1986) el algoritmo no emite salida, tolerando esta condición; para esta simulación se ajustó el detector a 80 A (Elneweihi et al., 1992). Durante la falla, se tiene un valor apreciable de corriente de secuencia negativa $I_{2}$ vista por el Rele A, permitiendo una detección y respaldo efectivo. En la última secuencia, el disparo de la línea 1 provocará una transferencia de potencia $I_{1}$ en el Rele A. Esta condición debe ser tolerada por el relevador, permitiendo la alimentación a la carga. El ajuste del detector de fallas simétricas fue similar al de un relevador de sobrecorriente convencional (ANSI/IEEE Std 141-1986, 1986). Durante esta condición, la salida del detector de falla propuesto no está presente, tendiendo un desempeño satisfactorio.

Con el incremento de sensibilidad (reducción de la corriente de arranque), el tiempo de operación del relevador de sobrecorriente se reduce. El efecto se ilustra en la figura 2.

Este comportamiento es benéfico especialmente en el tiempo de operación de la protección de respaldo, pero existe un riesgo de pérdida de coordinación. Una solución es la adaptación del tiempo de operación.
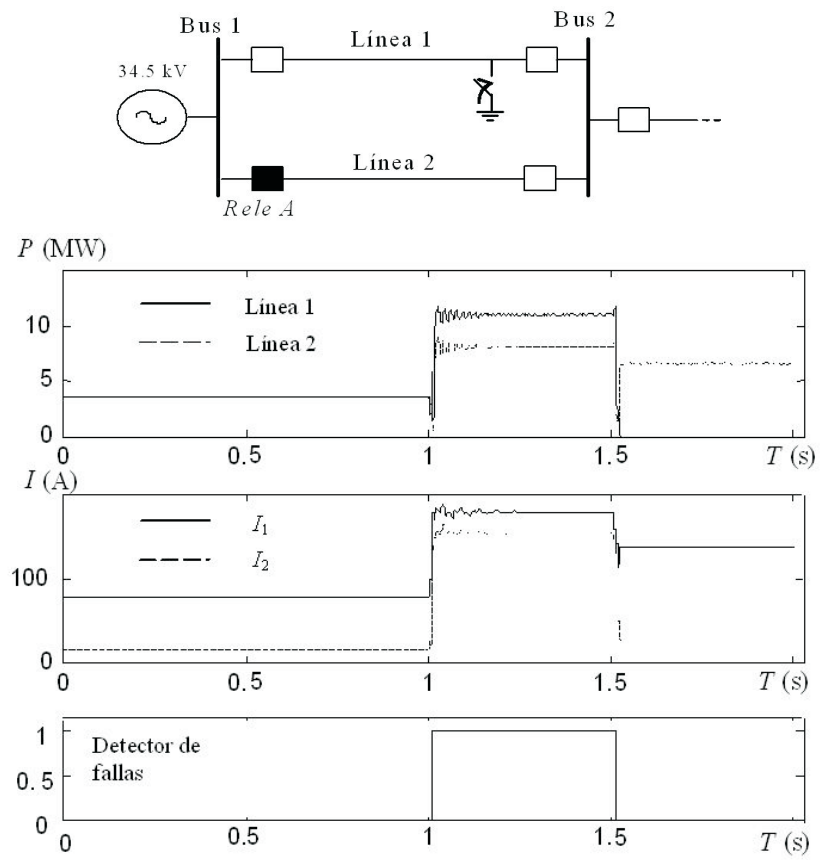

Figura 1. Evaluación de la lógica de detección de falla propuesta

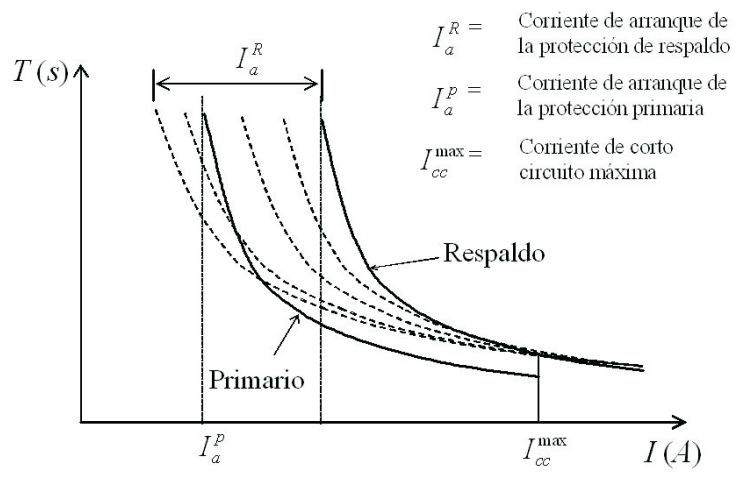

Figura 2. Efecto de la corriente adaptiva de arranque en el tiempo de operación 


\section{Tiempo adaptivo de operación}

El criterio de coordinación para relevadores de sobrecorriente es establecer una secuencia de operación, de tal forma que el tiempo de operación del relevador de respaldo $T_{R}$ sea mayor por un tiempo predefinido, que el tiempo de operación del relevador primario $T_{p}$; este intervalo de tiempo de coordinación (CTI) tiene un valor entre 0.2 a $0.4 \mathrm{~s}$.

$$
T_{R}=T_{P}+C T I .
$$

El propósito del proceso de adaptación es determinar una función de tiempo adaptiva, la cual defina el tiempo de operación del relevador de respaldo con un retardo de tiempo constante, relativo a la protección primaria para cualquier valor de corriente de falla.

Debido a la corriente de carga, el tiempo de respaldo es incrementado para valores mínimos de corriente de falla, aunque ambos relevadores tengan la misma curva. Para obtener el mismo retardo (CTI) para cualquier valor de corriente de falla hay dos mecanismos: el primero, modificar la palanca de tiempo para cada valor de corriente de falla (curvas 1, 2 y 3 en figura 3). Segundo, en la figura 3 se observa que la curva 4 es similar a la curva de la protección primaria $\left(\right.$ Rele $\left._{P}\right)$. Debido a la diferencia en el ajuste de la corriente de arranque entre $\operatorname{Rele}_{P}$ y $R e$ $l e_{R}$, la curva de Rele $_{R}$ no puede ser obtenida por medio del ajuste de la palanca de tiempo de Rele ; sin embargo, esta curva es asintótica a la corriente de arranque de Re$l e_{p}$. Por tanto, si la función de tiempo $T_{R}$ se determina en base a la expresión analítica de la curva de tiempo de la protección primaria evaluada con la corriente de arranque de la misma protección más el intervalo de coordinación (figura 4), se tiene el cumplimiento de (2) para valores de falla constante y variable. El modelo de la curva de tiempo usado en la figura 4 es IEC Standard 255-4 (1976). Las expresiones analíticas ilustradas en la misma figura corresponden a la curva de tiempo establecida en los relevadores de sobrecorriente de tiempo inverso, bajo esta relación tiempo y corriente se establece la operación del relevador. Las curvas son el tipo muy inversa, y los parámetros $A$ y $n$ son presentados en IEC Standard 255-4 (1976).

La expresión adaptiva del relevador es obtenida de (2). La corriente de operación $\left(I^{p}\right)$ es determinada como la razón de la corriente de cortocircuito del sistema eléctrico $\left(I_{c c}\right)$ y la corriente de arranque de la protección primaria $\left(I^{p}=I_{c c} / I_{a}^{p}\right)$. La función de tiempo adaptiva es:

$$
H\left(I^{P}\right)=\frac{1}{T_{p}\left(I^{P}\right)+C T I} .
$$

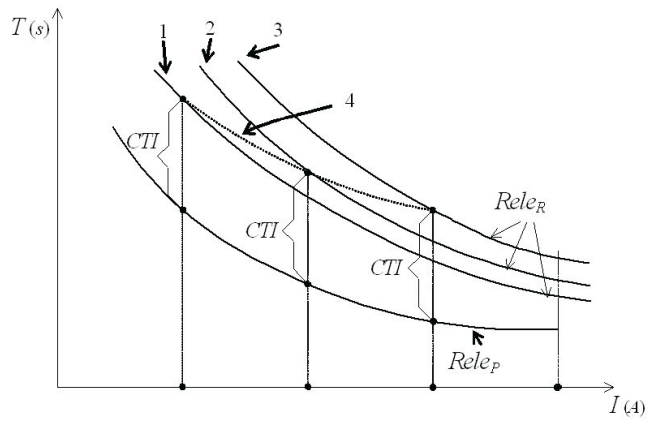

Figura 3. Característica de operación adaptiva

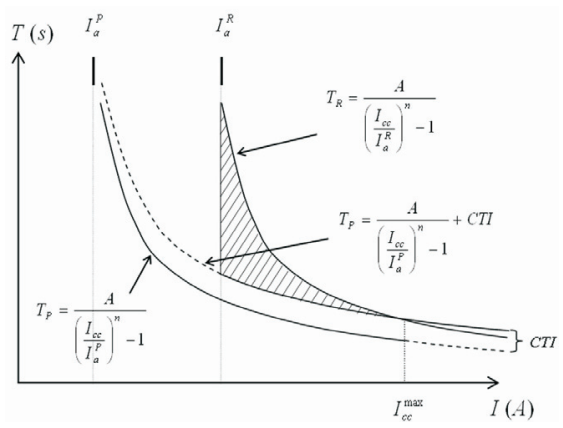

Figura 4. Curva de tiempo propuesta 
Cuando la característica de la protección primaria es conocida, $T_{p}\left(I^{P}\right)$ puede ser sustituida directamente en (3). Cuando la ecuación de tiempo es desconocida, pero la gráfica está disponible, es posible obtener pares de valores $(I, T)$ y usar un programa de ajuste de curvas fuera de línea para obtener $T_{P}\left(I^{P}\right)$ (Sachdev, 1978; Hieber, 1965; IEEE Standard C37.112-1996, 1996). Si la protección primaria es un fusible, únicamente es necesaria la curva de tiempo máximo de liberación; si la protección primaria es un restaurador, es necesaria la curva de tiempo y el ajuste de arranque.

La representación del estado dinámico del relevador de sobrecorriente utilizando la función adaptiva de tiempo es:

$$
\Delta t \sum_{k=1} H\left(I^{P}\right)=1
$$

donde $\Delta t$ es el periodo de muestreo, la condición de operación se cumple cuando el resultado de la integración de la función adaptiva $H\left(I^{P}\right)$ es igual la unidad (IEEE Standard C37.112-1996). El tiempo en el cual el relevador operó es determinado como el producto de la muestra que dio cumplimiento de la Ec. 4 y el periodo de muestreo.

Para condiciones de protección de líneas multi-terminales (Rele $A$ en la figura 1a), la característica de operación del relevador es definida por la protección primaria más lenta.

\section{Estructura funcional}

El diagrama funcional del relevador adaptivo de sobrecorriente propuesto es mostrado en la figura 5. El relevador adaptivo está compuesto básicamente de dos elementos adaptivos: corriente adaptiva de arranque para incrementar la sensibilidad del relevador; y tiempo adaptivo de operación para garantizar un retardo constante en la operación del relevador, independientemente de la magnitud de corriente de falla. La función principal del bloque de corriente adaptiva de arranque es la detección de falla. La corriente de arranque es determinada de (1) y restringida por el ajuste de un relevador convencional $I_{a}^{\max }$ y por la salida del relevador auxiliar 52a (ANSI/IEEE Std 141-1986), su salida es coherente con el estado del interruptor de potencia, de tal forma que al tener una salida $O$ es indicativo que la línea está puesta fuera de servicio y el ajuste del relevador adaptivo es $I_{a}^{\max }$. La señal $(T / F)$ es formada en la salida; si la falla ha sido detectada $\left(I_{k}>I_{a}\right)$ se emite la señal $T$ y la condición de arranque es declarada; se inicia entonces, el proceso de integración en el elemento de tiempo (4).

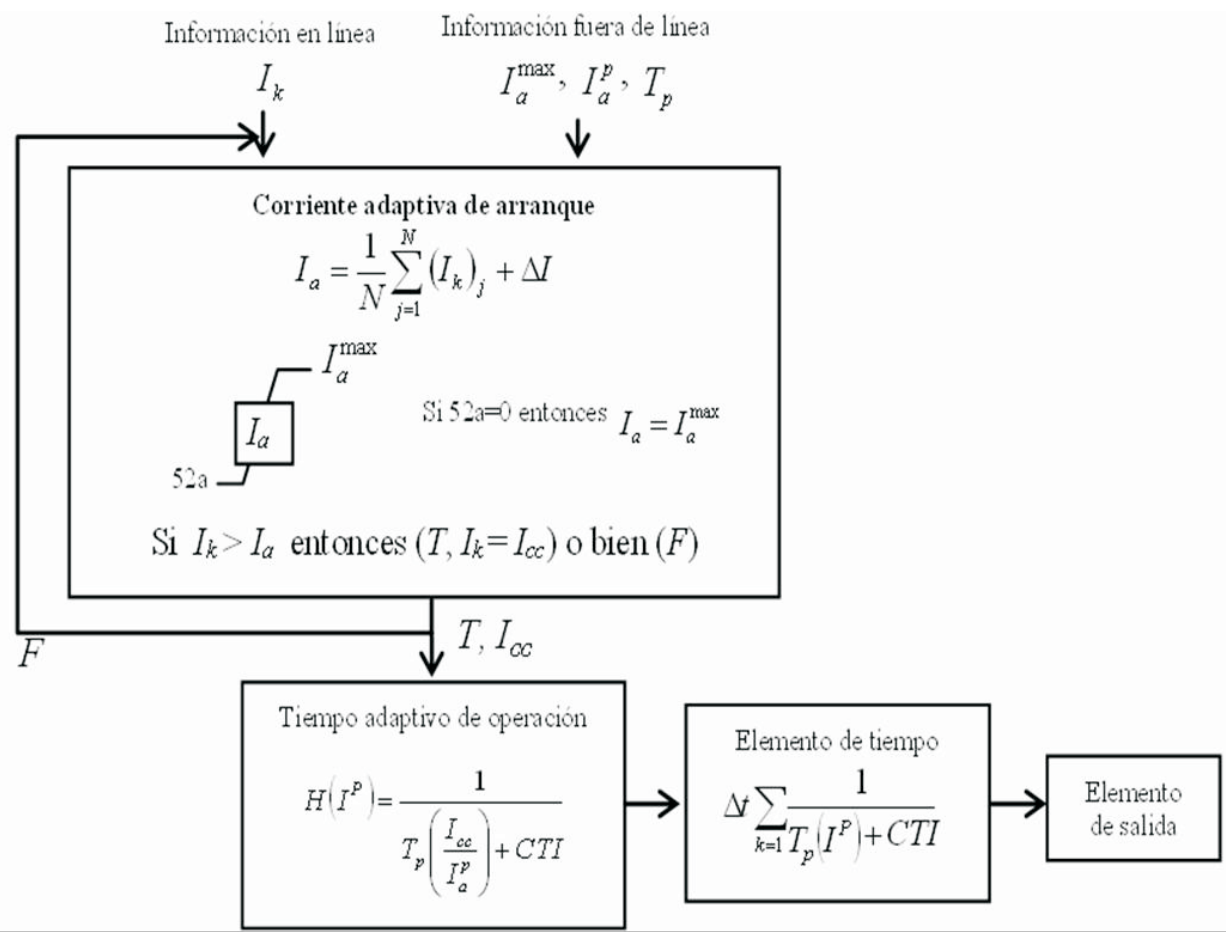

Figura 5. Diagrama funcional del relevador adaptivo de tiempo inverso 
DOI: http://dx.doi.org/10.22201/fi.25940732e.2010.11n2.013

Conde-Enriquez A., Vázquez-Martínez E. y Cantú-Gutiérrez V.P.

La señal $F$ es una condición de bloqueo para la operación del relevador.

El bloque de tiempo adaptivo de operación calcula el valor de la función $H\left(I^{P}\right)$ presentada en (4). La integración de $T_{P}$ emula la dinámica de operación de la protección primaria. El intervalo de tiempo de coordinación CTI (0.3 s) es adicionado para asegurar la coordinación entre el relevador adaptivo y el dispositivo primario. Si el elemento de corriente emite la señal permisiva $T$; la señal de salida del bloque de tiempo adaptivo es integrada por el elemento de tiempo. Este proceso de integración establece la dinámica de operación del relevador en presencia de corrientes de falla variables. Finalmente, en el elemento de salida, la condición de disparo es verificada y la señal de salida es enviada al circuito de disparo del interruptor.

El tiempo de operación del relevador adaptivo debe ser establecido como una función de la curva de tiempo del dispositivo primario. Existe, entonces, una independencia entre el tiempo de operación del relevador adaptivo (dependiente del tiempo de operación de la protección primaria), y la corriente de arranque (determinada según (1) y actuando únicamente como detector de fa1la). La corriente de arranque es dependiente de la condición de carga, permitiendo un incremento en la sensibilidad. Mediante el proceso de adaptación del tiempo de operación se obtiene una coordinación automática del relevador adaptivo con el dispositivo de protección primario; no es necesario realizar ningún procedimiento para su coordinación, aun cuando se presenten cambios en las condiciones de operación del sistema eléctrico, tales como: cambios topológicos, de generación y carga; que modifiquen la magnitud de la corriente de cortocircuito. Esto se observa en la figura 4 en donde al incrementarse el valor de corriente de cortocircuito el intervalo de coordinación según el criterio propuesto (4) se mantiene.

Durante una falla (trifásica o bifásica); la corriente de arranque del relevador adaptivo vigente es determinada en el periodo anterior del calculo de demanda, por tanto, la condición de falla es declarada y la función de tiempo es activada (figura 5).

Se presentan dos alternativas de operación en dependencia si la corriente de arranque del relevador adaptivo es menor a la corriente de arranque del relevador primario. En la primera alternativa, la corriente de arranque del relevador adaptivo tendrá un límite mínimo, la corriente de arranque del dispositivo primario; entonces, aun cuando el relevador adaptivo detecte la falla, el múltiplo de la corriente de arranque será menor que 1.0 y la operación no se efectuará. En la segunda alternativa, la corriente de arranque del relevador adaptivo no tendrá límite inferior, por tanto, será factible la operación del relevador adaptivo en condiciones en donde el dispositivo primario no tenga sensibilidad, esta condición no representa una pérdida de coordinación; la falla entonces, seria liberada por el relevador adaptivo.

Para efectuar el proceso de coordinación, es necesario obtener la expresión analítica de la curva de tiempo de la protección primaria, ya sea fusible, relevador electromecánico o restaurador, incluyéndola en la expresión dinámica del relevador adaptivo. No es necesario remover los dispositivos de protección existentes. La presencia del relevador adaptivo propuesto ofrece mayores posibilidades de solucionar problemas de sensibilidad y coordinación entre dispositivos de protección.

La coordinación entre una serie de relevadores de sobrecorriente (relevador adaptivo entre relevadores convencionales) se presenta en Conde (2003). La coordinación entre una serie de relevadores adaptivos es efectuada respetando el principio descrito, cada relevador tendrá la expresión analítica del relevador primario más CTI. Este proceso no implica tiempos elevados de operación para la serie de relevadores, porque el efecto de la corriente de carga es minimizado. La $I_{a}^{\max }$ para cada relevador es determinada en forma similar que en relevadores convencionales.

\section{Comparación entre relevadores de sobrecorriente}

En esta sección se presenta un estudio comparativo de sensibilidad y tiempo de operación entre tres tipos de relevadores de sobrecorriente: convencional, secuencia negativa y adaptivo.

\section{Análisis de sensibilidad}

El análisis de sensibilidad fue realizado en el sistema radial mostrado en la figura 6a. La corriente de falla mínima es simulada en el Bus 4. La variación de las impedancias $Z_{3}$ y $Z_{4}$, simulan una configuración multi-terminal y una variación de la corriente de carga, respectivamente. Para este análisis, los relevadores de sobrecorriente de fase fueron ubicados en el Bus 2 (Rele B). La sensibilidad es ponderada de acuerdo a la siguiente relación:

$$
\text { Sensibilidad }=\frac{I_{c c}^{\min }}{I_{a}} .
$$

Los métodos para determinar los valores de sensibilidad para el relevador convencional y el de secuencia negativa son descritos en Elneweihi et al. (1992) y 
ANSI/IEEE Std 141-1986 (1986), respectivamente. Para el relevador adaptivo, se asume que su corriente de arranque (1) no está limitada por el ajuste de arranque del dispositivo primario. Este es un estudio basado en un sistema en particular, las condiciones del estudio pueden cambiar, debido a la dependencia topológica, estados operativos y esquemas de protección; pero es posible obtener un estudio cualitativo de sensibilidad de los tres tipos de relevadores de sobrecorriente.

La corriente de falla mínima es determinada como una falla bifásica en condiciones de generación mínima y ubicada en el Bus 4. El valor aceptado de sensibilidad es 1.5 (ANSI/IEEE Std 141-1986,1986). En la figura 6b se muestran los valores de sensibilidad de los relevadores para diferentes valores de corriente de carga $\left(I_{k}\right)$ y contribuciones de la fuente de generación $\left(\mathrm{P}_{G e n}\right)$. Se observa que los relevadores convencional (plano 1) y de secuencia negativa (plano 2) son insensibles al cambio de demanda y mantienen la misma sensibilidad, debido a que su corriente de arranque es independiente de la dinámica de la carga.

En cambio, el relevador adaptivo tiene un ajuste que es dependiente de la dinámica de la carga, esto resulta en un incremento de la sensibilidad del relevador durante condiciones de demanda mínima comparado con los otros relevadores (ver plano 3 en figura $6 b$ ).

Durante condiciones de mayor contribución de potencia $\left(P_{G e n}\right)$ en la red, la sensibilidad de los tres tipos de relevadores analizados se incrementa (mayor corriente de falla). En el relevador adaptivo se presenta un incremento mayor de sensibilidad comparada con los otros relevadores.

Es posible concluir, que el relevador adaptivo presenta mayor sensibilidad que los relevadores convencionales y de secuencia negativa durante condiciones de demanda mínima y durante estados operativos de mayor contribución de potencia, debido a su mayor dependencia a la dinámica de la corriente de carga y a la variación del estado operativo (contribución de potencia).

La sensibilidad del relevador de secuencia negativa es similar al del relevador adaptivo, únicamente en demanda máxima y sólo para fallas bifásicas. La sensibilidad del relevador adaptivo siempre es mayor que la del relevador convencional.

En líneas multi-terminales (figura 6c), el relevador de secuencia negativa tiene mayor sensibilidad que el

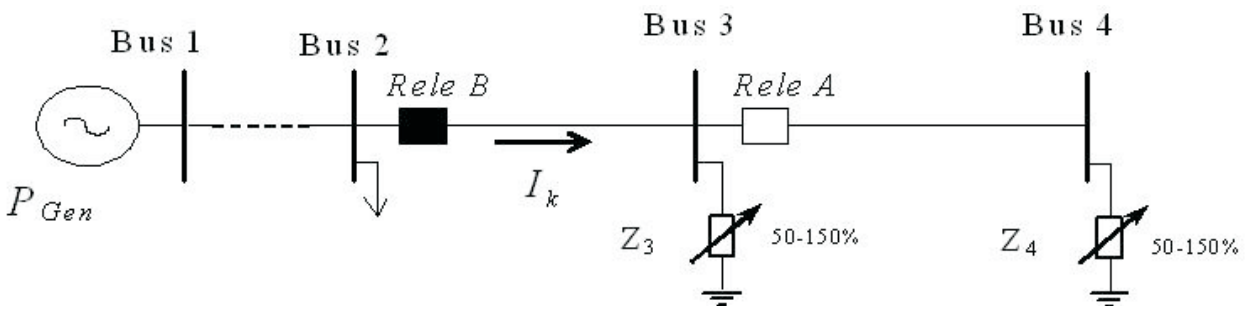

Figura 6a. Sensibilidad de relevadores de sobrecorriente de fase

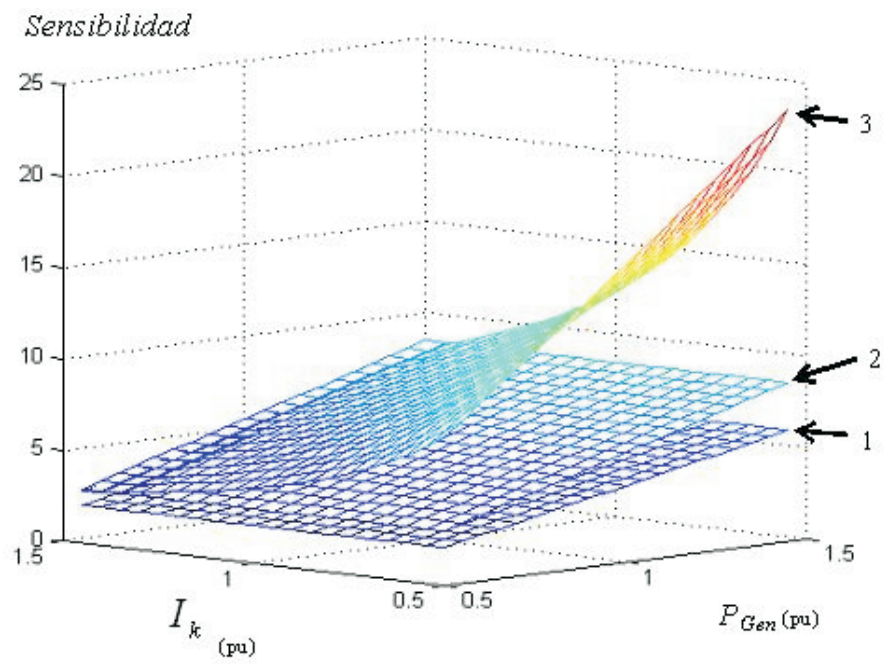

Figura 66. Sensibilidad de relevadores de sobrecorriente de fase 


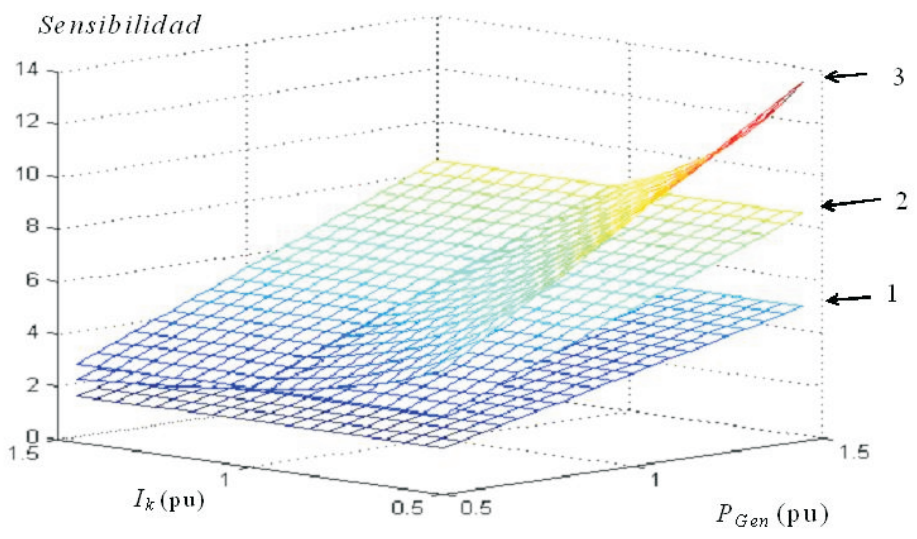

Figura 6c. Sensibilidad de relevadores de sobrecorriente de fase

relevador convencional, debido a que no es afectado por la corriente de carga, permitiendo definir su corriente de arranque un tanto mayor que la protección más lenta de los alimentadores de la configuración multi-terminal. Durante condiciones de máxima demanda, la sensibilidad de los relevadores de secuencia negativa es mayor que la de los relevadores restantes; sin embargo, bajo condiciones de demanda mínima, la sensibilidad del relevador adaptivo es la mayor.

De los resultados obtenidos podemos concluir que el relevador convencional es el que presenta menor sensibilidad, el relevador de secuencia negativa es más sensible en líneas multi-terminales (pero sólo para fallas bifásicas). El relevador adaptivo en cambio, tiene una mayor sensibilidad en redes radiales; y en redes multi-terminal, tiene mayor sensibilidad que el relevador de secuencia negativa en demanda mínima, cuando el relevador más lo necesita.

\section{Análisis de tiempo de operación}

El análisis de tiempo de operación fue efectuado en el sistema radial de la figura 6a. La corriente de arranque fue determinada por $Z_{3}$ y $Z_{4}$, ambos ajustados al $100 \%$. La figura 7 muestra el resultado del análisis de tiempo de los relevadores de sobrecorriente de fase. Se observa que el tiempo de respaldo del relevador de secuencia negativa es menor que el del similar convencional. El relevador adaptivo presenta un retardo constante (CTI), independientemente de la magnitud de la falla, esto resulta en tiempos menores de respaldo que los relevadores restantes.

Si la corriente de falla es menor que la corriente de arranque de la protección primaria, el relevador adaptivo podrá detectar la falla (lógica de detección de falla), por tanto, la corriente de arranque del relevador adaptivo que realiza funciones únicamente de detección de falla, puede ser activada y definir, como en los relevadores convencionales, el tiempo de operación. Este criterio puede ser opcional al usuario. La activación de este criterio ofrece una solución a la pérdida de sensibilidad de la protección primaria, sobre todo, en condiciones de demanda mínima. La curva de tiempo del relevador adaptivo será la misma que la de la protección primaria sin el CTI. Para la activación de esta lógica se tendrán que cumplir las condiciones de detección de falla (señal $T$ en figura 5) y el cumplimiento de la condición $I_{c c}<I_{a}^{P}$. Para el ejemplo mostrado en la figura 7 , el relevador primario tiene $300 \mathrm{~A}$ como ajuste de $I_{a}^{P}$, y el relevador adaptivo tiene $220 \mathrm{~A}$ como el mínimo ajuste para condiciones de demanda mínima.

Para cualquier falla entre 220-300A, el relevador adaptivo tendrá mayor sensibilidad que el relevador primario, por lo tanto, la falla a través de alta impedancia será detectada.

En la figura 8, el efecto combinado del relevador propuesto es mostrado. El relevador modifica su corriente de arranque según (1), este valor limita la longitud de la curva de tiempo; se tendrá entonces un comportamiento dinámico de la curva de tiempo dependiendo del valor vigente de la corriente de arranque.

Durante la demanda máxima el relevador adaptivo será aun más sensible que el relevador convencional, debido a que su ajuste será $15 \%$ mayor que la corriente de carga comparado con el 1.5 o 2.0 veces, de un relevador convencional.

El tiempo de respaldo (4) será sólo el CTI mayor que el tiempo de operación del primario para cualquier valor de corriente de falla. Por tanto, la sensibilidad y el tiempo de operación del relevador de sobrecorriente son mejorados mediante cambios dinámicos de ajuste. 


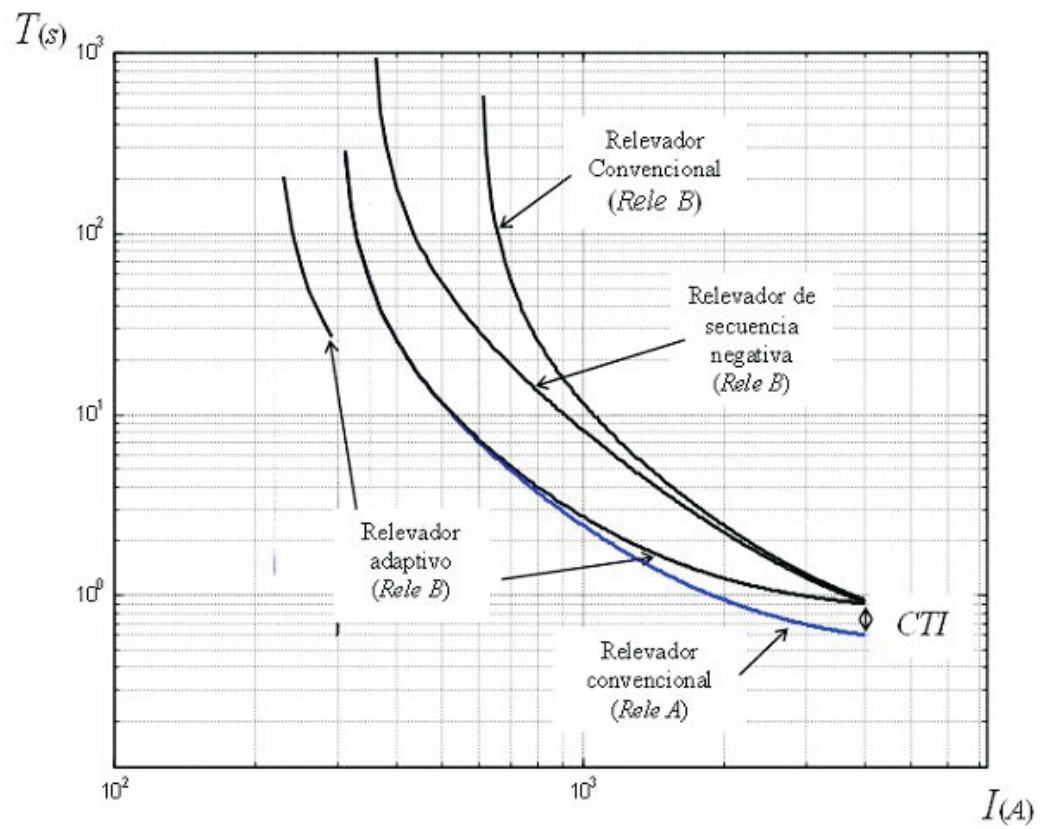

Figura 7. Comparación de curvas de tiempo de relevadores de sobrecorriente

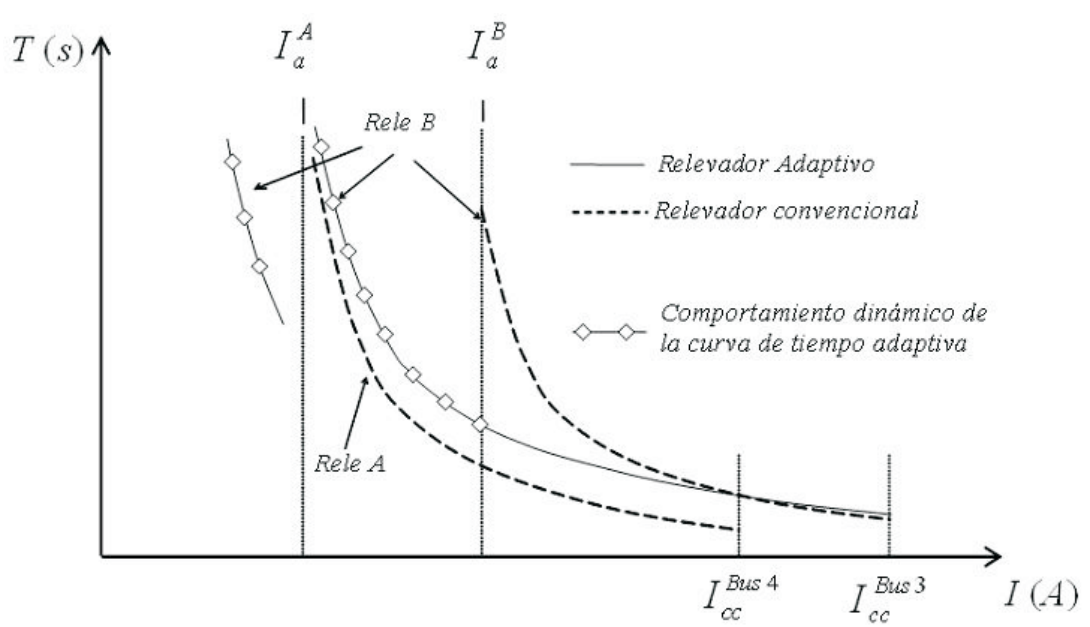

Figura 8. Comportamiento resultante del relevador adaptivo

\section{Conclusiones}

Los criterios adaptivos propuestos, incrementan la sensibilidad y reducen los tiempos de respaldo en los relevadores de sobrecorriente de tiempo inverso, principalmente durante escenarios de poca demanda.

Los parámetros de corriente de arranque y tiempo de operación son parámetros independientes en el relevador adaptivo. La corriente de arranque propuesta, incrementa la sensibilidad del relevador de sobrecorriente al ser dependiente de la corriente de carga vigente; esta corriente de arranque adaptiva funciona como un detector de falla, dando la señal permisiva para el inicio del proceso de integración de tiempo de operación del relevador adaptivo. El tiempo de operación del relevador adaptivo es determinado emulando la dinámica de operación del dispositivo primario (corriente de arranque y curva de tiempo del dispositivo primario) y adicionando el intervalo de coordinación, garantizando una optima coordinación.

Para incrementos apreciables de la corriente de carga, el relevador adaptivo tiene una lógica de detección 
DOI: http://dx.doi.org/10.22201/fi.25940732e.2010.11n2.013

Conde-Enriquez A., Vázquez-Martínez E. y Cantú-Gutiérrez V.P.

de fallas para supervisar la corriente de arranque. Esta lógica incluye una verificación de secuencia negativa y secuencia positiva. Esta lógica discrimina entre incrementos de carga y fallas (simétricas o asimétricas). Con la adaptación del tiempo de operación en el relevador de sobrecorriente, ademas de reducir los tiempos de respaldo, se obtiene una coordinación automática e independiente de cambios futuros en el sistema (tales como topología, generación y carga). El desempeño de la lógica de detección de falla fue satisfactorio, debido a que logra discriminar e identificar escenarios operativos tales como desbalance en estado estable, cortocircuito bifásico y disparo de la línea fallada (transferencia de potencia).

El relevador propuesto no requiere información de la red eléctrica adicional al de un relevador convencional, la rutina adaptiva es ejecutada únicamente con los fasores de corriente, esto exime de un incremento en la capacidad de hardware del relevador. Las funciones adaptivas propuestas son implementadas únicamente en el software del relevador. La simplificación algorítmica es obtenida con el uso del concepto de demanda en la definición de la corriente de arranque, requiriendo actualización cada 5 minutos; también el proceso de ajuste de curvas es fuera de línea, sin carga computacional al relevador. Entonces, el proceso adicional para el relevador adaptivo es mínimo. De las observaciones anteriores el relevador propuesto es obtenido con únicamente un ligero cambio en el "firmware" del relevador sin costo adicional.

\section{Referencias}

Abdelaziz A.Y., Talaat H.E.A., Nosseir A.I., Ammar A.H. An Adaptive Protection Scheme for Optimal Coordination of
Overcurrent Relays. Electric Power Systems Research, 61(1): 1-9, 28 February 2002.

ANSI/IEEE Std 141-1986. IEEE Recommended Practice for Electric Power Distribution for Industrial Plants.

Askarian H. et al. A New Optimal Approach for Coordination of Overcurrent Relays in Interconnected Power Systems. IEEE Transactions on Power Delivery,18(2), April 2003.

Conde A., Vázquez E., Altuve H.J. Time Overcurrent Adaptive Relay. International Journal of Electrical Power \& Energy Systems. 25(10):841-847. 2003.

Elneweihi A.F., Schweitzer E.O.III, Feltis M.W. Negative-Sequence Overcurrent Element Application and Coordination in Distribution Protection. IEEE Power Engineering Society. PES Summer Meeting, Seattle, WA, (July):12-16. 1992.

Hieber J.E. Empirical Equations of Overcurrent Relay Curves for Computer Application. IEEE Winter Power Meeting. New York, N.Y., January/February 1965, Paper No. 31 CP 65-91, pp. 1-11.

IEC Standard 255-4, Single Input Energizing Measuring Relays with Dependent Specified Time. IEC Publication 255-4. First Edition. 1976.

IEEE Standard C37.112-1996. IEEE Standard Inverse-time Characteristic Equations for Overcurrent Relays.

Sachdev M.S., Singh J., Fleming R.J. Mathematical Models Representing Time-Current Characteristics of Overcurrent Relays for Computer Application. IEEE Paper A78:131-5, January 1978.

Urdaneta A.J. et al. Optimal Coordination for Direccional Overcurrent Relays in Interconnected Power Systems. IEEE Transactions on Power Delivery, 3(3):903-911, July 1988.

\section{Semblanza de los autores}

Arturo Conde-Enríquez. Se graduó como ingeniero mecánico electricista en la Universidad Veracruzana en 1993. Obtuvo la maestría en ciencias de la ingeniería eléctrica y el grado de doctor en ingeniería eléctrica en la Universidad Autónoma de Nuevo León en 1996 y 2002, respectivamente. Actualmente es profesor investigador del programa doctoral de ingeniería eléctrica de la FIME-UANL y es miembro del sistema nacional de investigadores.

Ernesto Vázquez-Martínez. Se graduó como ingeniero en electrónica y comunicaciones en 1988, recibió su grado de maestro en ciencias de ingeniería eléctrica y su doctorado en ingeniería eléctrica por la Universidad Autónoma de Nuevo León, México, en 1991 y 1994, respectivamente. Desde 1996 trabaja como profesor investigador en el programa doctoral de ingeniería eléctrica de la UANL. Es miembro del IEEE y del sistema nacional de investigadores.

Vicente Paz Cantú-Gutiérrez. Se graduó de ingeniero electricista en 1977 y obtuvo su maestría en ciencias de la ingeniería eléctrica con especialidad en potencia en 1993 en la Facultad de Ingeniería Mecánica y Eléctrica de la Universidad Autónoma de Nuevo León, México, donde es profesor desde 1974. Actualmente desempeña el cargo de Coordinador del Departamento de Potencia Eléctrica. Sus áreas de investigación son la protección de sistemas eléctricos de potencia, y calidad de la energía. 